\title{
Impact of Intravesical Protrusion of the Prostate in the Treatment of Lower Urinary Tract Symptoms/Benign Prostatic Hyperplasia of Moderate Size by Alpha Receptor Antagonist
}

\author{
Yu Mi Seo, Hyung Jee Kim \\ Department of Urology, Dankook University College of Medicine, Cheonan, Korea
}

\begin{abstract}
Purpose: To evaluate whether intravesical protrusion of the prostate (IPP) is related to the treatment effect of alpha-1 receptor antagonist in patients with lower urinary tract symptoms (LUTS)/benign prostatic hyperplasia (BPH) with a prostate size of less than $40 \mathrm{~g}$.

Methods: A total of $77 \mathrm{BPH}$ patients over 50 years of age treated with alfuzosin (alpha blocker) were enrolled prospectively. The study included only patients with $\mathrm{BPH}$ of $40 \mathrm{~g}$ or less. The patients were classified into two groups depending on the presence of IPP at baseline: the IPP group (41 patients) and the non-IPP group (36 patients). Prostate volume, prostate-specific antigen (PSA), International Prostate Symptom Score and quality of life (IPSS/QoL), maximum flow rate (Qmax), and postvoid residual (PVR) volume were compared between the groups. The clinical significance of IPP was evaluated after the patients had been taking alfuzosin for 8 weeks.

Results: PSA and IPSS (total and voiding subscore) showed significant correlations with IPP $(\mathrm{P}<0.05)$. Comparison of parameters before and after 8 weeks showed that alfuzosin improved the total IPSS and all subscores $(\mathrm{P}<0.001), \mathrm{QoL}(\mathrm{P}<0.001)$, Qmax $(\mathrm{P}<0.001)$, and PVR $(\mathrm{P}=0.030)$ in the non-IPP group.

Conclusions: Alfuzosin may be less effective in improving symptom scores, PVR, and Qmax in the treatment of LUTS/BPH in the presence of IPP.
\end{abstract}

Keywords: Prostate; Lower urinary tract symptoms; Prostatic hyperplsia; Urinary bladder neck obstruction; Adrenergic alpha-1 receptor antagonists

\section{INTRODUCTION}

Lower urinary tract symptoms (LUTS)/benign prostatic hyperplasia $(\mathrm{BPH})$ develop through benign prostatic enlargement and bladder outlet obstruction [1]. Prostate enlargement does not occur homogeneously, however. Prostatic protrusion into the bladder often occurs and is a result of morphological changes [2]. Chia et al. [3] suggested that intravesical prostatic protrusion (IPP) is caused by the enlarging lateral lobes and the median lobe, and that the protrusion of the enlarged lobes causes a "ball-valve" type of obstruction, thus disrupting the funneling effect of the bladder neck and causing dyskinetic movement of the bladder during voiding. Thus, it is suggested that a prostatic mass with greater protrusion causes more severe voiding dysfunction by causing more serious bladder outlet obstruction [46]. It was also recently reported that increased IPP can affect the storage symptoms caused by stimulation of the bladder $[7,8]$.

According to previous studies, IPP is significantly correlated with increased prostate volume, increased transitional zone volume, greater obstructive symptoms, decreased maximum flow rate (Qmax), and increased postvoid residual (PVR), which suggests that IPP may have clinical usefulness in predicting the need for treatment [9].

It was also recently reported that tamsulosin may be more ef-
Corresponding author: Hyung Jee Kim

Department of Urology, Dankook University College of Medicine,

119 Dandae-ro, Dongnam-gu, Cheonan 330-997, Korea

Tel: +82-41-550-6630 / Fax: +82-41-551-6630

E-mail: killtumor@dankook.ac.kr

Submitted: November 1, 2012 / Accepted after revision: December 13, 2012
This is an Open Access article distributed under the terms of the Creative Commons Attribution Non-Commercial License (http://creativecommons.org/licenses/by-nc/3.0/) which permits unrestricted non-commercial use, distribution, and reproduction in any medium, provided the original work is properly cited. 
fective in improving symptom scores and Qmax in patients with mild IPP than in those with moderate or severe IPP. It was suggested that the larger the IPP, the worse the voiding symptoms and the less the response to treatment with alpha blockers [10]. If so, other treatment methods should be applied as soon as possible. However, in the study mentioned above, the measurement of IPP was performed retrospectively and the study population was small. Also, the study included patients with moderate and severe IPP and large prostate sizes. As a result, the effects of treatment with an alpha blocker were somewhat reduced. For these reasons, well-designed studies are needed to assess the effect of IPP on treatment.

In the present study, therefore, we assessed the effect of IPP on the treatment effect of alpha-1 receptor antagonists in patients with $\mathrm{BPH}$ of $40 \mathrm{~g}$ or less. We divided the patients into two groups depending on the presence or absence of IPP at baseline and compared urinary symptoms, Qmax, and residual urine volume between the groups. The clinical significance of IPP was evaluated after the patients had been taking alfuzosin for 8 weeks.

\section{MATERIALS AND METHODS}

This study was performed under Institutional Review Board approval. A total of 77 LUTS/BPH patients over 50 years of age treated with alfuzosin were enrolled prospectively. The study included only patients with BPH of $40 \mathrm{~g}$ or less. The patients were classified into two groups depending on the presence of IPP at baseline: the IPP group (41 patients) and the non-IPP group (36 patients). After the completion of urinalysis and routine laboratory tests at the first visit, prostate volume, prostatespecific antigen (PSA), International Prostate Symptom Score and quality of life (IPSS/QoL), Qmax, and PVR volume were compared between the groups. The clinical significance of IPP was evaluated by comparing the IPSS/QoL, Qmax, and PVR after the patients had been taking alfuzosin (10 $\mathrm{mg}$, daily) for 8 weeks.

Qmax was measured by uroflowmetry (Duet Logic G2, Mediwatch Plc, West Palm Beach, FL, USA), prostate volume and IPP were measured by transrectal ultrasonography ( $6.5 \mathrm{MHz}$; SA-8000, Medison, Seoul, Korea), and PVR was evaluated by bladder scan (Cubescan Biocon-500, Mcube Technology, Seoul, Korea). If the PSA of the patients was elevated above $4 \mathrm{ng} / \mathrm{mL}$, transrectal ultrasound-guided biopsies were performed to rule out prostate cancer, and only patients not diagnosed with pros- tate cancer were enrolled. Inclusion criteria were as follows: LUTS/BPH (less than $40 \mathrm{~g}$ ) diagnosed by ultrasound, persistent LUTS/BPH for more than 6 months, IPSS $\geq 12$, IPSS storage subscore $\geq 4$ and voiding subscore $\geq 5$, outpatient, and aged 50 years and older. A Qmax of less than $15 \mathrm{~mL} / \mathrm{sec}$ was an inclusion criterion. Patients with a medical history of urethral stricture, PVR $\geq 200 \mathrm{~mL}$, urinary tract infection, urinary tract stone disease, pelvic surgery, prostatic cancer, or neurogenic bladder or those taking anticholinergic agents were excluded at the start of evaluation. IPP was evaluated as measured by Nose et al. [11]. Length of IPP was obtained by measuring the vertical distance from the tip of the protrusion to the circumference of the bladder at the base of the enlarged prostate by transrectal ultrasound instead of the transabdominal approach of Nose et al. [11]. IPSS/ QoL scores were obtained for all patients, and IPSS scores were subdivided according to storage symptoms subscore and voiding symptoms subscore.

The primary endpoint was whether alfuzosin improved symptoms in the IPP group compared with the non-IPP group after medication for 8 weeks. The secondary endpoints were whether alfuzosin improved QoL, Qmax, and PVR in the IPP group compared with the non-IPP group after medication for 8 weeks.

All values are presented as the mean \pm standard deviation. The significance of differences among groups was determined by using Student's t-test with differences considered significant at $\mathrm{P}<0.05$.

\section{RESULTS}

The mean ages of the IPP and non-IPP groups were $67.2 \pm 6.9$ and $64.5 \pm 8.1$ years, respectively $(\mathrm{P}=0.125)$. The prostatic volumes of the IPP and non-IPP groups were $33.3 \pm 6.2 \mathrm{~mL}$ and $31.2 \pm 6.1 \mathrm{~mL}$, respectively $(\mathrm{P}=0.276)$, at baseline. The mean length of IPP in the IPP group was $6.1 \pm 2.2 \mathrm{~mm}$. The total PSA level was higher in the IPP group than in the non-IPP group at baseline. PSA and IPSS (total and voiding symptoms subscore) also differed significantly between the groups at baseline $(\mathrm{P}<$ 0.05). However, the storage symptoms subscore, QoL, PVR, and Qmax were not significantly different between the groups (Table 1).

Comparison of parameters before and after 8 weeks of medication showed that alfuzosin improved the total IPSS and all subscores $(\mathrm{P}<0.001)$, QoL $(\mathrm{P}<0.001)$, $\mathrm{Qmax}(\mathrm{P}<0.001)$, and $\mathrm{PVR}(\mathrm{P}=0.030)$ more effectively in the non-IPP group than in the IPP group. The improvements in the total IPSS score in the 
Table 1. Clinical data at baseline

\begin{tabular}{lccc}
\hline Parameter & IPP group & Non-IPP group & P-value \\
\hline No. & 41 & 36 & \\
Age $(\mathrm{yr})$ & $67.2 \pm 6.9$ & $64.5 \pm 8.1$ & 0.125 \\
tPSA $(\mathrm{ng} / \mathrm{mL})$ & $1.61 \pm 1.1$ & $1.04 \pm 0.48$ & 0.013 \\
tPV $\left(\mathrm{cm}^{3}\right)$ & $33.3 \pm 6.2$ & $31.2 \pm 6.1$ & 0.276 \\
IPSS & & & \\
$\quad$ Total & $22.2 \pm 4.9$ & $18.4 \pm 4.6$ & 0.004 \\
$\quad$ Voiding & $14.4 \pm 3.8$ & $11.8 \pm 3.6$ & 0.014 \\
$\quad$ Storage & $7.9 \pm 2.7$ & $6.5 \pm 3.8$ & 0.068 \\
Quality of life & $4.4 \pm 1.0$ & $4.2 \pm 0.9$ & 0.351 \\
Postvoid residual & $65.5 \pm 51.3$ & $47.3 \pm 43.9$ & 0.255 \\
Maximum flow rate & $8.9 \pm 2.8$ & $9.9 \pm 3.9$ & 0.424 \\
\hline
\end{tabular}

Values are presented as mean \pm standard deviation.

IPP, intravesical protrusion of the prostate; tPSA, total prostate-specific antigen; tPV, total prostate volume; IPSS, International Prostate Symptom Score.

IPP and non-IPP groups were $28.7 \pm 11.9 \%$ and $50.6 \pm 20.3 \%$, respectively $(\mathrm{P}<0.0001)$. In the non-IPP group, the total IPSS significantly improved after 8 weeks of medication. The patterns of change in QoL and in the storage and voiding symptoms subscores were similar.

Qmax by uroflowmetry improved more in the non-IPP group than in the IPP group after 8 weeks of medication $(\mathrm{P}<0.001)$. Changes in PVR showed a similar pattern (Table 2).

\section{DISCUSSION}

Theoretically, if voiding symptoms are the main symptoms in LUTS/BPH, an alpha-1 receptor antagonist is considered to be a treatment of first choice. Similarly, if storage symptoms are the main symptom, combined therapy with an alpha-1 receptor antagonist and an anticholinergic can be an appropriate choice. However, not all LUTS/BPH is managed satisfactorily. IPP may be one of the causes of such unsatisfactory management.

IPP is caused by enlargement of the lateral lobes and the median lobe; the protrusions of these enlarged lobes cause voiding symptoms [3]. Recently, there have been many reports about IPP. IPP is a useful predictor for evaluating the success of a voiding trial after acute urinary retention [12]. However, most such reports focused on the length of IPP and voiding symptoms. A few studies reported on the relation between IPP and storage symptoms $[7,8]$ or between IPP and clinical effects after alpha blocker medication [10]. In the study mentioned above, how-
Table 2. Clinical data after 2 months of alfuzosin $(10 \mathrm{mg})$ treatment

\begin{tabular}{lccc}
\hline Parameter & IPP group & Non-IPP group & P-value \\
\hline IPSS improvement (\%) & & & \\
$\quad$ Total & $28.7 \pm 11.9$ & $50.6 \pm 20.3$ & 0.0001 \\
Voiding & $29.2 \pm 13.8$ & $54.4 \pm 19.6$ & $<0.0001$ \\
Storage & $24.2 \pm 22.0$ & $41.6 \pm 28.0$ & 0.03 \\
QoL improvement (\%) & $24.8 \pm 19.8$ & $44.2 \pm 19.7$ & 0.001 \\
PVR improvement (\%) & $12.3 \pm 77.0$ & $44.4 \pm 36.3$ & 0.02 \\
Qmax imrovement (\%) & $30.5 \pm 33.8$ & $68.7 \pm 51.8$ & 0.0006 \\
\hline
\end{tabular}

Values are presented as mean \pm standard deviation.

IPP, intravesical protrusion of the prostate; IPSS, International Prostate Symptom Score; QoL, quality of life; PVR, postvoided residual; Qmax, maximum flow rate.

ever, the measurement of IPP was performed retrospectively, and the study population was small. As shown in previous reports, IPP is significantly correlated with increased prostate volume, transitional zone volume, greater obstructive symptoms, decreased Qmax, and increased PVR [9]. Therefore, with an increase in IPP, Qmax is decreased and PVR is increased. As a result, the IPP group showed less of a decrease in the QoL score after 8 weeks of medication than did the non-IPP group [7]. In addition, most previous studies included all sizes of $\mathrm{BPH}$ and thus large IPP. In the present study, we prospectively included only patients who prostate size was smaller than $40 \mathrm{~g}$. Our aim was to compare the differences in LUTS and in the effectiveness of an alpha blocker in patients with LUTS/BPH of similar prostate size according to the presence or absence of IPP.

In this study, baseline age, prostate volume, QoL, PVR, and Qmax did not differ significantly between the groups. Total PSA and IPSS (total and voiding subscore), however, did differ significantly between the groups at baseline $(\mathrm{P}<0.05)$. Total PSA was higher in the IPP group than in the non-IPP group, but the total PSA values of both groups were in the normal range and thus the difference was probably not significant clinically. However, compared with the IPSS after treatment, the percentage improvement was higher in the non-IPP group than in the IPP group. In the non-IPP group, all parameters were significantly improved at the endpoint of the study compared with the IPP group. Unlike in previous studies, however, IPP was not significantly related to increased prostatic volume, decreased Qmax, or increased PVR. These results imply that the prostate volume in this study was relatively small compared with previous studies. However, as previous studies have reported, IPP was related 
to worsening of voiding symptoms. Also, medication with alfuzosin yielded a better response in the non-IPP patient group. Furthermore, objective measurements such as changes in Qmax and PVR improved more in the non-IPP group than in the IPP group. Qmax by uroflowmetry was significantly improved in the non-IPP group after 8 weeks of medication. This result suggests that prostatic volume in this study was relatively small compared with previous studies.

The results of the present study suggest that because IPP is not related to initial IPSS, Qmax, or PVR, other study such as pressure-flow study is still needed for evaluation of bladder outlet obstruction. However, the presence of IPP implies a reduced effect of alpha blockers in the treatment of LUTS/BPH.

Our study did have some limitations. Even though it was a prospective study, the study population was small, and the enrolled patients did not undergo pressure-flow study. Despite these problems, however, the results of the present study suggest that the presence of IPP may indicate the need for a change in a therapeutic plan with further medical or surgical treatment.

In conclusion, even though prostate size was similar in the groups with and without IPP, treatment with alfuzosin for LUTS/ $\mathrm{BPH}$ was less effective in improving symptom scores, PVR, and Qmax in the presence of IPP.

\section{CONFLICT OF INTEREST}

No potential conflict of interest relevant to this article was reported.

\section{ACKNOWLEDGEMENTS}

The present research was conducted by the research fund of Dankook University in 2011.

\section{REFERENCES}

1. Roehrborn CG. Benign prostatic hyperplasia: an overview. Rev Urol 2005;7 Suppl 9:S3-14.

2. Lee SW, Cho JM, Kang JY, Yoo TK. Clinical and urodynamic significance of morphological differences in intravesical prostatic pro- trusion. Korean J Urol 2010;51:694-9.

3. Chia SJ, Heng CT, Chan SP, Foo KT. Correlation of intravesical prostatic protrusion with bladder outlet obstruction. BJU Int 2003;91: 371-4.

4. Lim KB, Ho H, Foo KT, Wong MY, Fook-Chong S. Comparison of intravesical prostatic protrusion, prostate volume and serum prostatic-specific antigen in the evaluation of bladder outlet obstruction. Int J Urol 2006;13:1509-13.

5. Reis LO, Barreiro GC, Baracat J, Prudente A, D’Ancona CA. Intravesical protrusion of the prostate as a predictive method of bladder outlet obstruction. Int Braz J Urol 2008;34:627-33.

6. Kim BH, Sohn JC, Park CH, Kim CI. The usefulness of intravesical prostatic protrusion and bladder wall thickness measurement using transabdominal ultrasound in patients with benign prostatic hyperplasia. Korean J Urol 2005;46:1180-5.

7. Lee JM, Chung H, Kim TW, Kim HS, Wang JH, Yang SK. The correlation of intravesical prostatic protrusion with storage symptoms, as measured by transrectal ultrasound. Korean J Urol 2008;49:145-9.

8. Doo CK, Uh HS. Anatomic configuration of prostate obtained by noninvasive ultrasonography can predict clinical voiding parameters for determining BOO in men with LUTS. Urology 2009;73: 232-6.

9. Lieber MM, Jacobson DJ, McGree ME, St Sauver JL, Girman CJ, Jacobsen SJ. Intravesical prostatic protrusion in men in Olmsted County, Minnesota. J Urol 2009;182:2819-24.

10. Park HY, Lee JY, Park SY, Lee SW, Kim YT, Choi HY, et al. Efficacy of alpha blocker treatment according to the degree of intravesical prostatic protrusion detected by transrectal ultrasonography in patients with benign prostatic hyperplasia. Korean J Urol 2012;53:92-7.

11. Nose H, Foo KT, Lim KB, Yokoyama T, Ozawa H, Kumon H. Accuracy of two noninvasive methods of diagnosing bladder outlet obstruction using ultrasonography: intravesical prostatic protrusion and velocity-flow video urodynamics. Urology 2005;65:493-7.

12. Tan YH, Foo KT. Intravesical prostatic protrusion predicts the outcome of a trial without catheter following acute urine retention. J Urol 2003;170(6 Pt 1):2339-41.

13. Lee JM, Chung H, Kim TW, Kim HS, Wang JH, Yang SK. The correlation of intravesical prostatic protrusion with storage symptoms, as measured by transrectal ultrasound. Korean J Urol 2008;49:145-9. 